\title{
Contributed Papers for JD16: Abstracts
}

\section{Status of the International Celestial Reference Frame:}

Alan L. Fey (U.S. Naval Observatory, 3450 Massachusetts Avenue NW, Washington DC, 20392-5420, USA); Jean Souchay (Observatoire de Paris, DANOF, 61, avenue de l'observatoire, 75014 Paris, France)

\begin{abstract}
We present a brief report on the status of the International Celestial Reference Frame. There have been two extensions (updates) of the ICRF since its initial definition in 1998. The primary objectives of extending the ICRF were to provide positions for the 109 extra-galactic radio sources observed since the definition of the ICRF and to refine the positions of candidate and other sources using additional observations. A secondary objective was to monitor sources to ascertain whether they continue to be suitable for use in the ICRF. Positions of the ICRF defining sources have remained unchanged. Improved positions and errors for the candidate and other sources were estimated and reflect the changes in the data set and the analysis. The 109 new sources were added with ICRF coordinates. We also discuss current efforts toward ICRF maintenance and the International Celestial Reference System Product Center.
\end{abstract}

\section{Potential Refinement of the ICRF:}

Chopo Ma (NASA Goddard Space Flight Center, Code 926, Greenbelt, MD 20771, USA)

\begin{abstract}
The ICRF analysis and data represented the state of the art in global, extragalactic, X/S band microwave astrometry in 1995. Similar analysis has been used to extend the ICRF with subsequent data consistent with the original catalog. Since 1995 there have been considerable advances in the geodetic/astrometric VLBI data set and analysis that would significantly improve the systematic errors, stability, and density of the next realization of the ICRS when the decision is made to take this step. In particular, data acquired since 1990, including extensive use of the VLBA, are of higher quality and astrometric utility because of changes in instrumentation, schedule design, and networks as well as specifically astrometric intent. The IVS (International VLBI Service for Geodesy and Astrometry) continues a systematic extension of the astrometric data set. Sufficient data distribution exists to select a better set of defining sources. Improvements in troposphere modeling will minimize known systematic astrometric errors while accurate modeling and estimation of station effects from loading and nonlinear motions should permit the re-integration of the celestial and terrestrial reference frames with Earth orientation parameters though a single VLBI solution. The differences between the current ICRF and the potential second realization will be described.
\end{abstract}

\section{The CRF Solution by the Geoscience Australia IVS Analysis Center:} Oleg Titov (GPO Box 378, Canberra, ACT, 2601, Australia)

Abstract. The Geoscience Australia IVS Analysis Center is working for improvement of the ICRF. The catalogue of 659 radio-source positions estimated from a 23-year set of the VLBI data (1980-2003) in a homogeneous solution 
(TRF, CRF, EOP) has been obtained using the OCCAM software. Statistical analysis of the results shows that the median accuracy of the source positions is 0.2-0.3 mas in both coordinates. The long-term apparent motions of two quasars: $0923+392(4 \mathrm{C} 39.25)$ and $2145+067$ are also analyzed.

\title{
Contribution of stable sources to ICRF improvements:
}

A.-M. Gontier \& Martine Feissel-Vernier (Observatoire de Paris, SYRTE, 61, avenue de l'observatoire, 75014 Paris France)

\begin{abstract}
A set of stable compact radio sources is proposed for the future maintenance of the ICRF axes, based on time series analysis of VLBI-derived coordinates of extragalactic radio sources. The selection scheme makes a combined use of statistical and deterministic tests. It identifies 199 stable sources, to be compared to the current 212 defining sources. Their consideration for the maintenance of the frame axes is expected to improve the frame stability by a factor of five, reaching the level of less than 10 microarcseconds in the medium term. The improved stability and internal consistency of the frame also enhances the quality of the Earth orientation determinations.
\end{abstract}

\section{Astrometric Microlensing and Degradation of Reference Frames:}

Mizuhiko Hosokawa (Communications Research Laboratory, Tokyo, 184-8795, Japan); Kouji Ohnishi (Nagano National College of Technology, Nagano, 3818550, Japan); Toshio Fukushima (National Astronomical Observatory, Mitaka, Tokyo, 181-8588, Japan)

\begin{abstract}
We have pointed out that the positions of extragalactic radio sources fluctuate on the order of a micro arcsecond ( $\mu$ as) because of astrometric microlensing by stars and MACHOs in our galaxy (Hosokawa et al. 1997). This means that the kinematical reference frames based on the positions of extragalactic radio sources will be degraded by this kind of fluctuation. Recently, we have shown that in the case of the extragalactic sources in the direction close to the Galactic Center, the optical depth of $10 \mu$ as fluctuation is about a quarter for the standard model of our galaxy (Hosokawa et al. 2002). Further, macro scale astrometric gravitational lensing by the collective mass of the Galactic Center appears in a different manner. In this paper we discuss the type, the magnitude and the time scale of the degradation of reference frames due to the astrometric gravitational lensing. For the astrometric gravitational lensing event of a few $\mu$ as caused by each star, the event duration is expected to be several years. On the other hand, the induced motion due to macro scale astrometric gravitational lensing is practically regarded as secular.
\end{abstract}

\section{Extending the ICRF to Higher Radio Frequencies: 24 \& $43 \mathrm{GHz}$ :}

Christopher S. Jacobs (Jet Propulsion Laboratory, California Institute of Technology, 4800 Oak Grove Drive, Pasadena, CA 91109, USA); Patrick Charlot, David Gordon, Gabor Lanyi Chopo Ma, Charles Naudet, Ojars Sovers, Liwei Zhang, and the KQ VLBI Survey Collaboration

\footnotetext{
Abstract. Astrometric observations of active galactic nuclei have been used to construct quasi-inertial global reference frames, most notably the International Celestial Reference Frame (ICRF) which now forms the basis for all as-
} 
trometry including deep space navigation. The ICRF was defined using X- (8.4 $\mathrm{GHz})$ and $\mathrm{S}$-band $(2.3 \mathrm{GHz})$ observations collected over $20+$ years. There are several motivations for extending this work to higher frequencies, namely, to construct a more stable frame based on more compact sources, to provide calibrators for phase referencing, and to support spacecraft navigation at higher frequencies.

Survey observations using the Very Long Baseline Array at K-band (24 $\mathrm{GHz}$ ) and Q-band ( $43 \mathrm{GHz})$ have been undertaken to pursue these goals. Three observing sessions have covered the full 24 hours of right ascension and declination down to $-30 \mathrm{deg}$. The resulting catalog of $80+$ sources has K-band median formal position uncertainties of $<200$ micro-arcseconds. The Q-band positional uncertainties are about 1.5 times larger. Group delay residuals were excellent at approximately $20 \mathrm{psec}$ weighted RMS. Comparison of the K-band frame to the $\mathrm{S} / \mathrm{X}$-band ICRF shows systematic errors which we will discuss.

The research performed at JPL-Caltech and GSFC was done under contract with NASA.

\title{
Densification of the ICRF/HCRF in Visible Wavelengths:
}

Sean E. Urban (U.S. Naval Observatory, 3450 Massachusetts Ave. NW, Washington DC, 20392-5420, USA)

\begin{abstract}
With the release of the Hipparcos Catalogue and the IAU resolution designating its 100,000 star subset as the HCRF, the optical reference frame increased 20-fold in the number of stars over the FK5. For many applications, even these 100,000 stars are inadequate in spatial density and limiting magnitude. However, Hipparcos is dense enough and deep enough to reduce many astrometric catalogs directly; the resulting data can be used to densify the HCRF and extend its magnitude limit. The initial major densification efforts of the HCRF utilized the Tycho data, culminating in the Tycho-2 Catalogue of 2.5 million stars. Over the last few years, several groups have been working toward even greater densification. Some resulting catalogs, such as the CMC 13, M2000, and SPM 2.0 are zonal, covering selected areas of the sky. Others, such as UCAC, USNO-B, and GSC 2.3 aim at global coverage but with varying degrees of accuracy and magnitude regimes. Others, namely DENIS and 2MASS, are extending the HCRF into the near IR realm. The current state of many of these projects, and what is expected in the next few years in terms of densification of the optical frame, is presented.
\end{abstract}

Extending the ICRF into the Infrared: 2MASS-UCAC Astrometry: Norbert Zacharias (U.S. Naval Observatory, 3450 Massachusetts Ave. NW, Washington DC, 20392-5420, USA); Howard L. McCallon, Eugene Kopan \& Roc M. Cutri (IPAC, Caltech, MS 100-22, 770 S. Wilson Ave., Pasadena, CA 91125, USA)

Abstract. An external comparison between the infrared 2MASS and the optical UCAC positions was performed, both being on the same system, the ICRS. About 48 million sources in common were identified. Random errors of the 2MASS catalog positions are about 60 to 70 mas per coordinate for the $K_{S}$ $=4$ to 14 range, increasing to about 100 to 150 mas for saturated and very faint stars. Systematic position differences between the 2 catalogs are very small, about 5 to 10 mas as a function of magnitude and color, with somewhat larger 
errors as a function of right ascension and declination. The extension of the ICRF into the infrared has become a reality.

\title{
Progress on Linking Optical-Radio Reference Frames Using CCD Ground-Based Telescopes:
}

N.Maigurova, G.Pinigin, Yu.Protsyuk (Nikolaev Astronomical Observatory, Observatorna 1, Nikolaev, 54030 UKRAINE); R.Gumerov (Kazan State University, Kremlevskaya 18, Kazan, 420008 RUSSIA); Z.Aslan \& I.Khamitov (Turkish National Observatory TUG, Antalya, 07058 TURKEY); W.Jin, Z.Tang, S.Wang (Shanghai Astronomical Observatory, Nandan Road 80, Shanghai, 200030 CHINA)

\begin{abstract}
Results of the Joint Project between observatories from China, Russia, Turkey and Ukraine on improvement of linking optical and radio reference frames are discussed. The 300 extra-galactic radio sources (ERS) observation program is extended due to the increase of observations in the southern hemisphere up to $-40^{\circ}$ declination. At present, observations of more than 150 ERS were used for reduction. The intermediate internal estimation of the link between optical and radio reference frames showed values near zero within an accuracy of about 6 mas by using secondary reference stars from UCAC2. A comparison of presented results with those of other investigations was made.
\end{abstract}

\section{Extragalactic Source Structure:}

Patrick Charlot (Observatoire Aquitain des Sciences de l'Univers-CNRS/UMR 5804, BP 89, 33270 Floirac, France)

\begin{abstract}
The compact extra-galactic radio-emitting objects used to define the I nternational Celestial Reference Frame (ICRF) are only imperfect fiducial points in the sky on milliarcsecond scales. Many sources show frequency- and time-dependent extended emission structures with varied morphologies that are explained in the framework of unified theories of active galactic nuclei. Such theories are also useful to make predictions about the expected source morphology at optical and IR wavelengths. Various imaging surveys are now available to evaluate the source compactness and astrometric suitability on a statistical basis. Additionally, exploratory work is being conducted to correct for the effect of source structure in actual astrometric analysis by using hundreds of source maps, as available from ongoing structure monitoring. Recent progress in these areas are reviewed in the framework of future possible ICRF realizations.
\end{abstract}

\section{Geophysical Nutation Model:}

Véronique Dehant (Royal Observatory of Belgium, Ringlaan 3, B-1180 Brussels, Belgium)

\begin{abstract}
The nutation model that has been adopted by the IAU in 2000 is the semi-analytical model MHB2000 of Mathews et al. (2002, JGR 107(B4), DOI: $10.1028 / 2001 \mathrm{JB} 000390$ ). We show how robust this model is and examine the information about the interior of the Earth that has been derived. The observations used to derived the parameters of MHB2000 as well as the amplitude of the Free Core Nutation (FCN) are examined in terms of their stability and
\end{abstract}


precision. Additional contributions from the external geophysical fluids (atmosphere, ocean) are also studied and shown to be non-negligible.

Practical Consequences of the Improved Precession-Nutation Models: Patrick T. Wallace (Space Science \& Technology Dept., CLRC / Rutherford Appleton Laboratory, Chilton, Didcot, OX14 4HH, United Kingdom)

\begin{abstract}
The IAU 2000 precession-nutation models are classical in form, unlike the associated Earth rotation model. The counterpart of GST in the new system is "Earth Rotation Angle", which is directly proportional to UT1 and is reckoned with respect to a kinematically-defined "non-rotating origin", the CEO. Though essential for interpreting Earth-rotation at VLBI accuracies and simplifying the computation of $(h, \delta)$, the introduction of the CEO has aroused considerable controversy. The new models came into use for VLBI analysis at 2003 January 1 , and subsequent IERS Bulletins provide $(\delta X, \delta Y)$ relative to the IAU 2000 celestial pole in addition to the existing $(\delta \Delta \psi, \delta \Delta \epsilon)$ corrections to IAU1976/80. The almanac offices face interesting choices of how to present the new quantities and which, if any, of the existing tabulations can be omitted. Most astronomers will, however, be little affected. In parallel with developing the new canonical models, considerable efforts have been made also to provide classical equivalents of similar accuracy, to give users a choice of when (or whether) to adopt the new methods. The models, both new and classical, have been implemented as Fortran subroutines and published through the IERS website. An extended set of routines has been released through the IAU SOFA initiative.
\end{abstract}

\title{
IERS Conventions:
}

Dennis D. McCarthy (U.S. Naval Observatory, 3450 Massachusetts Ave. NW, Washington DC, 20392-5420, USA); Gerard Petit (Bureau International des Poids et Mesures, Paris, France)

\begin{abstract}
The International Celestial Reference Frame (ICRF) is currently a radio reference frame accessed through Very long Baseline Interferometry (VLBI) and refined with technique-dependent improvements described in this Joint Discussion. An important component of the International Celestial Reference System (ICRS) that is the basis for this frame is the set of conventional models and procedures that are used to define the system. The International Earth Rotation and Reference System Service (IERS) Conventions Center, provided jointly by the U.S. Naval Observatory (USNO) and the Bureau International des Poids et Mesures (BIPM), produces the IERS Conventions that contain the models and procedures needed to realize and access the ICRS. The key elements of the Conventions related to the ICRS are outlined, and recent improvements are
\end{abstract}


highlighted. Improvements in the IERS Conventions (models and procedures) should play a role by globally improving IERS products.

\title{
New Precession Formula:
}

Toshio Fukushima (NAO Japan, 2-21-1, Ohsawa, Mitaka, Tokyo, 181-8588, Japan)

\begin{abstract}
We adapted J.G. Williams' expression of the precession and nutation using the 3-1-3-1 rotation (Williams 1994) to an arbitrary inertial frame of reference. The modified formulation avoids a singularity caused by finite pole offsets near the epoch. By adopting the planetary precession formula numerically determined from DE405 (Harada and Fukushima 2003) and by using a recent theory of the forced nutation of the non-rigid Earth, SF2001 (Shirai and Fukushima 2001), we analyzed the celestial pole offsets observed by VLBI for 1979-2000 (McCarthy 2000, private communication) and determined the best-fit polynomials of the luni-solar precession angles. Then we translated the results into the classic precession quantities and evaluated the difference in them due to the difference in the ecliptic definition. The combination of these formulas and the periodic part of SF2001 serves as a good approximation of the precessionnutation matrix in the ICRF.
\end{abstract}

ICRS, ITRS, and the IAU Resolutions Concerning Relativity:

Mike Soffel \& Sergei Klioner (Lohrmann-Observatorium, Institut fur Planetare Geodasie, Technische Universitat Dresden, Mommsenstrasse 13, D-01062 Dresden, Germany)

\begin{abstract}
The IAU2000 Resolutions concerning relativity introduce two celestial reference systems, a barycentric one, BCRS with coordinates $(\mathrm{t}, \mathrm{x})$, and a geocentric one, GCRS with coordinates $(T, X)$. The two sets of coordinates are related by 4 -dimensional space-time transformations. So far the relations of the BCRS and GCRS with the ICRS and ITRS have NOT been discussed. This will be done here. It is argued that the ICRS is a special representation of the BCRS, and the ITRS-coordinates differ from the GCRS ones by a time dependent rotation of spatial coordinates plus possible scale factors. This implies that also the ICRS- and the ITRS- coordinates are related by a generalized Lorentz-transformation.
\end{abstract}

\section{Earth Orientation Catalogue - An Improved Reference Frame:}

Jan Vondrák \& Cyril Ron (Astronomical Institute, Academy of Sciences of the Czech Republic, Boční II, 14131 Prague 4, Czech Republic)

\begin{abstract}
Optical observations of latitude/universal time variations of nearly five thousand stars (made during the twentieth century at 33 observatories in Earth orientation programmes) are used, in combination with the Hipparcos and Tycho catalogues and their combination with ground-based observations (ARIHIP), to derive a new improved star catalogue called the Earth Orientation Catalogue (EOC). The first version of the catalogue, EOC-1, based on the observations with instruments observing in local meridian, is now finished. The improved proper motions and/or positions are more convenient for long-term extrapolation of apparent places than those of the original Hipparcos and Ty-
\end{abstract}


cho Catalogues. The catalogue EOC is planned to be used for another new re-analysis of Earth orientation parameters based on optical astrometry observations in the 20 th century.

\title{
Astrometry With Large Un-Astrometric Telescopes:
}

Imants Platais (Department of Physics and Astronomy, Johns Hopkins University, Baltimore, MD 21218, USA)

\begin{abstract}
Over the last few decades the number of large-sized telescopes has grown dramatically. These new telescopes were designed to produce sharp images of stars and galaxies and are not necessarily optimized for astrometric measurements. Despite this and some other limitations cutting-edge astrometry can be done with such instruments. Space-based CCD imagers can easily break the 1-mas precision limit, while ground-based telescopes are currently limited to accuracies of 3-5 mas. It is expected that the Large Synoptic Survey Telescope will produce a 15 petabyte imaging database down to $V=28$ and over 30,000 square degrees of the sky, which opens new horizons for astrometry.
\end{abstract}

\section{Status of Space-Based Astrometric Missions:}

Ralph Gaume (U.S. Naval Observatory, 3450 Massachusetts Ave. NW, Washington DC, 20392-5420, USA)

\begin{abstract}
Since the highly successful Hipparcos space-based astrometry mission, a number of follow-up programs have been proposed to accomplish a wide variety of scientific goals. Due in part to funding pressure and technical challenges, the status of these missions has changed on a nearly continuous basis (proposal, selection, descope, cancellation, rescope, reproposal). The status, capabilities, operational concept, science goals, schedules, and technical challenges of the current set of space-based astrometric missions will be discussed.
\end{abstract}

\section{Realization of the Inertial Frame with GAIA:}

François Mignard (OCA/CERGA, av. Copernic, F06130 Grasse, France)

\begin{abstract}
The European Space Agency astrometry mission Gaia scheduled for a launch in mid-2010 will observe thousands of extra-galactic sources together with nearly one billion stars. With a target accuracy of 10 microarcseconds at $V=15$ and 50 microarcseconds at $V=18$, this will open a new era in the realization of the primary reference frame. The very principles of this direct realization in the visible are outlined in this paper together with a discussion of modeling problems or intrinsic limitations due to the galactic rotation, source instability or the occurrence of gravitational lensing.
\end{abstract}

\section{Astrometric Goals of the RadioAstron Mission:}

Vladimir E. Zharov, Igor A. Gerasimov, Konstantin V. Kuimov (Sternberg State Astronomical Institute, 13, Universitetskij prospect, 119992 Moscow Russia); 
Aleksander E. Rodin \& Yury P. Ilyasov (Puschino Radio Astronomy Observatory, 142290 Puschino Russia)

\begin{abstract}
The high-apogee elliptical orbit of the RadioAstron international mission impacts precision astrometry on the level of about a microsecond of arc ( $\mu$ as). Optimal launch of the RadioAstron mission could be on March 2006. The RadioAstron program is the next generation of the radioastrometry programs. According the RadioAstron program, initiated by the Astro Space Center of Lebedev Physical Institute of Russian Academy of Science (supervisor N.S.Kardashev) in collaboration with other institutes of Russia and abroad, the satellite SPECTR carrying a 10-meter radio telescope will be launched in a highly eccentric orbit with eccentricity $e=0.853$. Apogee distance will be $350000 \mathrm{~km}$. It is planned that the VLBI observations will be conducted with the largest ground-based radio telescopes. The period of revolution of the satellite will be about 9.5 days, and observational time during one revolution can be about 9 days. Observations can be made at $\mathrm{P}, \mathrm{L}, \mathrm{C}$, and $\mathrm{K}$ frequency bands with a bandwidth of $32 \mathrm{MHz}$. For the shortest wavelength $1.35 \mathrm{~cm}$ and baseline of about $350000 \mathrm{~km}$ resolving power of order of $8 \mu$ as can be achieved. It is planned to solve both astrophysical and astrometry problems during a total expected lifetime of the satellite of three years. The main astrometric goal of the mission is the realization of a new International Celestial Reference Frame based on measurements of the coordinates of $\sim 100$ defining sources with microarcsecond accuracy. Observations of some pulsars on the space-ground interferometers and by the timing technique will allow us to connect kinematical and dynamical systems with unprecedented accuracy. Coordinates of ground radio telescopes will be determined with respect to the geocenter.
\end{abstract}

\title{
Misleading Proper Motions of Galactic Objects at the Microarc- second Level: \\ Jean Kovalevsky (Observatoire de la Cote d'Azur, Avenue Copernic, 06130 Grasse, France)
}

\begin{abstract}
At the microarcsecond (muas) level of accuracy of observations foreseen by the GAIA program, the effects of the curvature of stellar orbits around the center of the galaxy cannot be neglected. The curvature of the solar system barycentric motion induces a time dependent component of the aberration, which reaches, in some regions of the sky, 4 muas per year. In the case of stars, it is combined with a similar effect due to the curvature of the galactocentric orbit of the star, which may reach 600 muas per year for a star situated at 50 parsecs from the center of the galaxy, and much larger closer to it. The formulae permitting us to compute these apparent proper motions are given together with the maps describing this effect within the galaxy. They disappear if one uses a galactocentric rather than a barycentric reference frame. The advantages and difficulties of this frame are discussed.
\end{abstract}


Relating the Dynamical Reference Frame and the Ephemerides to the ICRF:

E. M. Standish (CalTech/Jet Propulsion Laboratory; 301-150; Pasadena, CA 91109, USA)

\begin{abstract}
In the past, the motions of solar system bodies were used to establish a non-rotating "inertial" reference system, often referred to as "the dynamical reference frame". With the establishment of the ICRF, the use of a dynamical reference frame has become obsolete in this context.

Planetary ephemerides, on the other hand, continue to play vital roles in a number of other applications, such as spacecraft navigation, mission design, etc. For present-day ephemerides, the accuracies of the inner planet positions are $1 \mathrm{~km}$ or less in all coordinates, including the orientation of the system onto the ICRF. Certain quantities are much better known over specific time intervals (e.g., Earth-Mars distance over the past few decades, throughout which it has been accurately measured). The outer planet positions are significantly less wellknown, being established during the present era by CCD observations to about 0 " 05 or so, but quickly deteriorating for decades away from the present.
\end{abstract}

\title{
The ICRS and the IERS Information System:
}

Wolfgang R. Dick, Bernd Richter, \& Wolfgang Schwegmann (IERS Central Bureau, BKG, Richard-Strauss-Allee 11, 60598 Frankfurt am Main, Germany)

\begin{abstract}
This paper discusses the role of the International Earth Rotation and Reference Systems Service (IERS) for the International Celestial Reference System (ICRS) with emphasis on the IERS Information System, consisting of data, publications, documentation, and other information in printed and online form. Plans for a new database-driven information system are outlined.
\end{abstract}

\section{ICRS - ITRS Connection Consistent with IAU (2000) Resolutions:}

Irina Kumkova (Institute of Applied Astronomy RAS, 10, Kutuzov emb, St.Petersburg, 191186 RUSSIA); Michael Stepashkin (Saint-Petersburg Institute for Informatics and Automatics RAS)

\begin{abstract}
Algorithms of direct and reverse relativistic 4-dimensional transformation of barycentric and geocentric celestial reference coordinate systems (BCRS and GCRS) according to IAU Resolution B1(2000) are developed. Transformation between BCRS and GCRS is considered as a part of general process of linking the International Celestial Reference System to the International Terrestrial Reference System, provided by the International Earth Rotation and Reference System Service.
\end{abstract}

\section{Reference Frames and Ground-Based Astrometry:}

Magda Stavinschi (Astronomical Institute of the Romanian Academy, Str. Cutitul de Argint 5, RO-040558 Bucharest, Romania, E-mail: magda@aira.astro.ro)

\footnotetext{
Abstract. We try to analyze the contribution to the extension of optical reference frame to faint stars in crowded fields, the production of input catalogues for future space projects, re-observation of existing catalogues for proper motion
} 
determination, link of optical and radio reference frames, position of radio-source optical counterparts, stellar catalogues, etc. It is one of the tasks of the WG on The Future Development of Ground-Based Astrometry.

The Future Development of Ground-Based Astrometry:

Magda Stavinschi (Astronomical Institute of the Romanian Academy, Str. Cutitul de Argint 5, RO-040558 Bucharest, Romania, E-mail: magda@aira.astro.ro); Jean Kovalevsky (Observatoire de la Côte d'Azur - CERGA, av. Copernic, F06130 Grasse, France, E-mail: Jean.Kovalevsky@obs-azur.fr)

Abstract. This WG was created following the decision of Division 1 at the XXIVth IAU GA at Manchester. During the three years it was chaired by M. Stavinschi (Romania) and Jean Kovalevsky (France). Members are Daffydd Wyn Evans (UK), Carlos Lopez (Argentina), Dan Pascu (USA), Antonio Pugliano (Italy), Manuel Sanchez (Spain), Ramakhrisna Teixeira (Brazil), and Arthur Upgren (USA).

\title{
Astrometric Measurements of Radio Sources Optical Counterparts. OATo Campaign: Some Final Results:
}

B. Bucciarelli, M.T. Crosta, M.G. Lattanzi, G. Massone, R. Morbidelli (Astronomical Observatory of Torino, I-10025, Italy); W. Jin, Z. Tang (Astronomical Observatory of Shanghai, 200030 China); G. Deiana, A. Poma, S. Uras (Astronomical Observatory of Cagliari, I-09012, Italy)

\begin{abstract}
We present photographically determined positions of a sub-set of 20 radio sources from a larger sample collected by OATo during a dedicated campaign, as described in this paper. Targets are from the list of extra-galactic objects adopted by the IAU for the realization and maintenance of the ICRF. The results obtained so far are in agreement with the VLBI-determined radio positions at a significance level of better than 100 microarcseconds.
\end{abstract}

\section{Coupling Between the Earth's Rotation Rate and Precession-Nutation:} Sébastien Lambert (SYRTE - UMR8630/CNRS, Observatoire de Paris, 61 avenue de l'Observatoire, 75014 Paris, France)

\begin{abstract}
For different Earth models, we computed the effects of variations in the Earth's rotation rate on precession-nutation. In the case of a refined model with elastic mantle and decoupled liquid core, we found a major contribution of $-136.56 \mu$ as and $6.18 \mu$ as on the 18.6-year nutation respectively in longitude and in obliquity, and a decrease of $-3222.80 \mu \mathrm{as} /$ century on the precession rate in longitude.
\end{abstract}

ICRF Densification Via HIPPARCOS-2MASS Cross-Identification: Goran Damljanović (Astronomical Observatory, Volgina 7, 11160 Belgrade, Ser- 
bia and Montenegro); Jean Souchay (Observatoire de Paris, DANOF, 61, avenue de l'observatoire, 75014 Paris, France)

\begin{abstract}
We developed a cross-identification programme of stars of any two catalogues, and applied it to HIPPARCOS and 2MASS. It found 37940 common stars, after selecting a rejection criterion, that we set to a $3 \sigma$ value. The procedure was more than $68.5 \%$ successful by using the data of catalogues. There were 117955 HIPPARCOS stars, and 162195232 2MASS Second Incremental Data Release ( $\sim 47 \%$ of the sky) ones in our basic selection. Then, we calculated a preliminary systematic error $\left(\sim 0^{\prime \prime} 10\right)$ of differences $\Delta \alpha$ and $\Delta \delta$ of detected common stars, included it into the programme, and the cross-identification was near $80 \%$ successful.
\end{abstract}

\title{
Extending the ICRF to Higher Radio Frequencies - First Imaging Re- sults:
}

A. L. Fey, D. A. Boboltz (U.S. Naval Observatory - 3450 Massachusetts Avenue NW, Washington DC, 20392-5420, USA); P. Charlot (Observatoire Aquitain des Sciences de l'Univers - CNRS/UMR 5804, BP 89, 33270 Floirac, France); E. B. Fomalont (National Radio Astronomy Observatory - 520 Edgemont Road, Charlottesville, Virginia 22903-2475, USA); G. E. Lanyi, L. D. Zhang (Jet Propulsion Laboratory - California Institute of Technology, 4800 Oak Grove Drive, Pasadena, CA 91109-8099, USA); and the K-Q VLBI Survey Collaboration

\begin{abstract}
We present first imaging results and preliminary source structure analysis of 108 extra-galactic objects observed using the Very Long Baseline Array (VLBA) at $24 \mathrm{GHz}$ and $43 \mathrm{GHz}$ as part of a joint NASA, USNO, NRAO and Bordeaux Observatory program to extend the ICRF to higher radio frequencies.
\end{abstract}

\section{Japan Astrometry Satellite Mission - The JASMINE Project:}

Taihei Yano, Naoteru Gouda, Yukiyasu Kobayashi, Takuji Tsujimoto, Yukitoshi Kan-ya (National Astronomical Observatory, Mitaka, Tokyo 181-8588, Japan); Yoshiyuki Yamada (Graduate School of Science, Kyoto University, Sakyo-ku, Kyoto 606-8502, Japan); Hiroshi Araki, Seiichi Tazawa, Kazuyoshi Asari,Seiitsu Tsuruta, Hideo Hanada, Nobuyuki Kawano (National Astronomical Observatory, Mizusawa, Iwate 023-0861, Japan)

\begin{abstract}
JASMINE is the name of a Japanese infrared (z-band: $0.9 \mu \mathrm{m}$, or $\mathrm{K}$-band: $2.2 \mu \mathrm{m}$ ) scanning astrometric satellite, planned to be launched between 2013 and 2015. The main objective of JASMINE is to study the fundamental structure and evolution of the disk and the bulge components of the Milky Way Galaxy. Its important objective is to investigate stellar physics. In order to accomplish the objective, JASMINE will measure parallaxes, positions and proper motions with a precision of 10 microarcsec ( $\mu$ as) at $\mathrm{z}=15.5 \mathrm{mag}$ or $\mathrm{K}=12 \mathrm{mag}$.
\end{abstract}

\section{The USNO Extragalactic Reference Frame Link Program:}

Marion I. Zacharias (USRA, 1101 17th St. NW, Suite 1004, Washington DC, USA and U.S. Naval Observatory, 3450 Mass. Ave. NW, Washington DC, 20392, 
USA); Norbert Zacharias, \& Theodore J. Rafferty (U.S. Naval Observatory, 3450

Mass. Ave. NW, Washington DC, 20392, USA)

\begin{abstract}
The objective of this project is to improve the link between the radio and optical reference frames over the initial Hipparcos effort. The alignment of the radio-optical systems is important for absolute proper motions and source identification across the wavelength spectrum. There are 717 ICRF +extensions radio sources. The U.S. Naval Observatory (USNO) extra-galactic reference frame link program includes all applicable $(\approx 600)$ sources with optical counterparts. Furthermore, USNO is involved in the extra-galactic link of the Space Interferometry Mission (SIM) and plans for a new astrometric, ground-based, 1-meter class telescope.
\end{abstract}

\title{
Testing the Hipparcos/ICRF Link Using Radio Stars:
}

D. A. Boboltz, A. L. Fey, K. J. Johnston, N. Zacharias \& R. A. Gaume (U.S. Naval Observatory, 3450 Massachusetts Avenue NW, Washington DC, 203925420 , USA)

\begin{abstract}
We have undertaken an effort to verify and improve the radio/optical frame link through connected element interferometer astrometry of radio stars. In our first epoch, we observed 19 radio stars using the Very Large Array in A-configuration plus the Pie Town Very Long Baseline Array antenna $(\mathrm{VLA}+\mathrm{PT})$. Differences in right ascension and declination were computed between our VLA+PT data and the Hipparcos positions updated to our epoch (2000.94). A weighted least-squares fit to the position differences was performed and the three rotation angles between the radio and optical frames were determined. We found that the two frames are aligned to within the formal errors of approximately 3 milliarcseconds per axis. Presented here are the results of this work and the plans for future work.
\end{abstract}

\section{Another Look at Non-Rotating Origins:}

George H. Kaplan (U.S. Naval Observatory, 3450 Massachusetts Ave. NW, Washington DC, 20392-5420, USA)

\begin{abstract}
Two "non-rotating origins" were defined by the IAU in 2000 for the measurement of Earth rotation: the Celestial Ephemeris Origin (CEO) in the ICRS and the Terrestrial Ephemeris Origin (TEO) in the ITRS. Universal Time (UT1) is now defined by an expression based on the angle $\theta$ between the $\mathrm{CEO}$ and TEO. Many previous papers, e.g., Capitaine, Guinot, \& McCarthy (2000), developed the position of the CEO in terms of a quantity $s$, the difference between two arcs on the celestial sphere. A similar quantity $s^{\prime}$ was defined for the TEO.

As an alternative, a simple vector differential equation for the position of a non-rotating origin on its reference sphere is presented here. The equation can be easily numerically integrated to high precision. This scheme directly yields the unit vector of the CEO in the ICRS, or that of the TEO in the ITRS, as a function of time. This simplifies the derivation of the main transformation matrix between the ITRF and the ICRS. The directness of the development may
\end{abstract}


have pedagogical and practical advantages for the vast majority of astronomers who are unfamiliar with the history of this topic.

\title{
IDV Sources as ICRF Sources: Viability and Benefits:
}

Roopesh Ojha (Australia Telescope National Facility, CSIRO, PO Box 76, Epping, NSW 1710, Australia); Alan L. Fey (U.S. Naval Observatory, 3450 Massachusetts Ave. NW, Washington DC, 20392-5420, USA); David L. Jauncey (Australia Telescope National Facility, CSIRO, PO Box 76, Epping, NSW 1710, Australia); Kenneth J. Johnston (U.S. Naval Observatory, 3450 Massachusetts Ave. NW, Washington DC, 20392-5420, USA); James E. Lovell \& Lucyna Kedziora-Chudczer (Australia Telescope National Facility, CSIRO, PO Box 76, Epping, NSW 1710, Australia)

\begin{abstract}
Radio sources that exhibit rapid variability in their light curves, as a result of radio wave propagation through turbulent electron density fluctuations in the interstellar medium, appear to be the most compact sources in the sky. In particular, the most variable weak sources, might be the most point-like and, thus, some of the best candidates for densification of the International Celestial Reference Frame (ICRF) and consequent improvement in its accuracy. Further, the advent of the Mk IV/V VLBI system will make use of weaker sources easier. We will discuss the viability of this idea and state the benefits that might flow from this approach.
\end{abstract}

\section{USNO/ATNF Astrometry and Imaging of Southern Hemisphere ICRF Sources:}

Roopesh Ojha, David L. Jauncey (Australia Telescope National Facility, CSIRO, PO Box 76, Epping, NSW 1710, Australia); Alan L. Fey, Kenneth J. Johnston (U.S. Naval Observatory, 3450 Massachusetts Ave. NW, Washington DC, 20392-5420, USA); Richard G. Dodson, Simon D. Ellingsen, Peter M. McCulloch (University of Tasmania, Hobart, Australia); George D. Nicolson, Jonathan F. H. Quick (Hartebeesthoek Radio Astronomy Observatory, Krugersdorp, South Africa); John E. Reynolds, Anastasios K. Tzioumis \& Warwick E. Wilson (Australia Telescope National Facility, CSIRO, PO Box 76, Epping, NSW 1710, Australia)

\begin{abstract}
The USNO and the ATNF are collaborating in a continuing VLBI research program in southern hemisphere source imaging and astrometry. The goals of this program are two fold: to image all southern hemisphere ICRF sources at least twice for structure monitoring and to search for new astrometric sources for densification of the ICRF. All 184 existing ICRF sources south of -20 degrees, have been observed for imaging at least once. Imaging and a second epoch $\mathrm{g}$ observations are underway. In order to identify new extra-galactic radio sources to be added to the ICRF, survey observations of selected ATCA calibrator sources have been interspersed among the imaging observations. These survey observations have, to date, identified a total of 29 possible astromet-
\end{abstract}


ric targets. Dedicated astrometric VLBI experiments have been scheduled to determine accurate positions for these sources.

\title{
CCD-Based Astrometric Measurements of Photographic Plates:
}

I. H. Bustos Fierro \& J. H. Calderon (Observatorio Astronomico, Universidad Nacional de Cordoba, Rep. Argentina)

\begin{abstract}
A methodology for the astrometric measurement of photographic plates making use of a scientific grade CCD camera was developed and tested on a Carte du Ciel plate. In order to measure a complete $\mathrm{CdC}$ plate a mosaic of $\mathrm{f} 64$ frames with partial overlap in both coordinates was made. With the aim of evaluating the accuracy of stellar centroids a MAMA-based digitization of the same plate was employed as a pattern. We found a noticeable radial distortion produced by the optical system of the camera that was corrected. The reduction to celestial coordinates was performed by means of the blockadjustment technique, using Tycho-2 as a reference catalog. Differences with Tycho-2 suggest that the errors of CCD-based positions obtained ftom the CdC plate are between 0.20 and 0.25 arcseconds. These positions are intended to be employed in the determination of proper motions at few mas/yr level, therefore allowing densification of the proper motion system in regions of interest of the sky up to photographic magnitude 14.5 (for CdC plates), using a relatively low cost device available at our own observatory.
\end{abstract}

\section{Relativistic Satellite Attitude: Joining Local and Global Reference Frames for the Realization of Space-Borne Astrometric Catalogues:}

Donato Bini (Istitute "M. Picone", C.N.R., and I.C.R.A., Rome, Italy); Beatrice Bucciarelli, Maria T. Crosta, Mario G. Lattanzi, and Alberto Vecchiato (Turin Astronomical Observatory, Pino Torinese, Turin, Italy); Fernando de Felice (Department of Physics, University of Padova, Padova, Italy)

\footnotetext{
Abstract. The definition of the satellite rest frame plays a fundamental role in the relativistic treatment of space observations. We find a mathematical representation of this frame which is an analytical solution accurate to $(v / c)^{3}$ and corresponds to the expected attitude of the astrometric satellite Gaia. We named this frame attitude frame since it takes into account the satellite's actual orbital motion and attitude law, both computed in global BCRS coordinates. Moreover, we show that the running time on Gaia is compatible with IAU resolution B1.5 (2000).
} 